\title{
A Luteinizing Hormone-based Protocol Versus Traditional Flexible Gonadotropin-Releasing Hormone Antagonist Protocol in Women with Normal Ovarian Response: Study Protocol for a non- Inferiority Trial
}

Ya-su Lv

Beijing Chaoyang Hospital

Yuan Li

Beijing Chaoyang Hospital

Shan Liu ( $\sim$ liushan821009@126.com )

Beijing Chao-Yang Hospital: Beijing Chaoyang Hospital https://orcid.org/0000-0001-8056-556X

Study protocol

Keywords: randomized controlled trial, in-vitro fertilization, luteinzing hormone, cumulative ongoing pregnancy rate

Posted Date: June 14th, 2021

DOI: https://doi.org/10.21203/rs.3.rs-76107/v1

License: (c) (i) This work is licensed under a Creative Commons Attribution 4.0 International License. Read Full License 


\section{Abstract}

\section{Background}

Use of gonadotropin-releasing hormone $(\mathrm{GnRH})$ antagonists during the late follicular phase can prevent premature luteinizing hormone $(\mathrm{LH})$ surge. Many patients demonstrate an insufficient endogenous LH concentration during ovarian stimulation. Previous studies have demonstrated that ultra-low LH concentration influences pregnancy outcomes. However, affected patients cannot be distinguished prior to ovarian stimulation using baseline characteristics alone. With traditional fixed or flexible GnRH antagonist protocols, antagonist administration may further reduce LH activity. Previously, we proved that LH can be used as an indicator for the timing and dosage of antagonist. Patients with a persistently low LH concentration during ovarian stimulation may not require antagonists, whereas antagonist administration can affect reproductive outcomes. To further explore this hypothesis, we designed a randomized clinical trial to compare the LH-based flexible $\mathrm{GnRH}$ antagonist protocol with traditional flexible $\mathrm{GnRH}$ antagonist protocol in women with normal ovarian response.

\section{Methods}

This study was a multicenter, parallel, prospective, randomized, non-inferiority study. The primary efficacy endpoint was cumulative ongoing pregnancy rate per cycle. The study aimed to prove the non-inferiority of cumulative ongoing pregnancy rate per cycle with a LH-based flexible $\mathrm{GnRH}$ antagonist protocol versus traditional flexible $\mathrm{GnRH}$ antagonist protocol. Secondary endpoints were the high-quality embryo rate, clinical pregnancy rate, and cancellation rate. Differences in cost-effectiveness and adverse events were evaluated. The cumulative ongoing pregnancy rate per cycle in women with normal ovarian response was $70 \%$. Considering that a non-inferiority threshold should retain $80 \%$ of the clinical effect of a control treatment, a minimal clinical difference of $14 \%$ (one-sided: $a, 2.5 \% ; \beta, 20 \%$ ) and a total of 338 patients were needed. Anticipating a $10 \%$ dropout rate, the total number of patients required was 372 .

Discussion

This is the first randomized controlled trial to compare the efficacy of a LH-based treatment regimen with a traditional flexible $\mathrm{GnRH}$ antagonist protocol during ovarian stimulation. We hypothesized no significant difference in cumulative ongoing pregnancy rate per cycle between the two protocols. Moreover, patients with insufficient endogenous LH during ovarian stimulation may benefit from LHbased $\mathrm{GnRH}$ antagonist protocols. The results will provide new information on when to introduce antagonists and the appropriate dosage of antagonist.

Trial registration: ClinicalTrials.gov, ChiCTR1800018077. Registered on 29 August, 2018.

\section{Background}


There is evidence that a sufficient concentration of luteinizing hormone $(\mathrm{LH})$ is necessary for normal follicular growth and oocyte maturation. A low LH concentration during ovarian stimulation can adversely affect follicular morphology, quality, and maturation, determining meiotic status and fertilization ability $[1,2]$. Extensive clinical trials have shown that a serum $L H$ concentration of $\geq 1.2 \mathrm{IU} / \mathrm{L}$ is necessary to provide adequate LH support to follicle-stimulating hormone (FSH)-induced follicular development [2-4]. Some studies have demonstrated that for optimal cyclic follicular development, the serum LH concentration should be between $1.2 \mathrm{IU} / \mathrm{L}$ and $5.0 \mathrm{IU} / \mathrm{L}$ [4-6]. Other studies suggest that the indications for use of LH with gonadotropin-releasing hormone $(\mathrm{GnRH})$ antagonists during ovarian follicular development are the mid-follicular period (day 6), an estradiol (E2) concentration of $<200 \mathrm{pg} / \mathrm{ml}$, no follicles $>10 \mathrm{~mm}$ in size, an endometrial thickness of $<6 \mathrm{~mm}$, and a baseline serum LH concentration of $<1.2 \mathrm{IU} / \mathrm{ml}$ on day $6[6,7]$. However, only a small number of studies have been performed, and there are no clear guidelines with regard to the optimal serum LH concentration or supplementation time; thus, these are areas worthy of further study.

Although FSH can induce follicular growth in the absence of $\mathrm{LH}$, follicles may have developmental defects, such as abnormally reduced E2 production and a lack of lutealization and rupture capacity on the trigger day [8,9]. GnRH antagonist protocols have been widely used for ovarian stimulation. Use of GnRH antagonists during the late follicular phase can prevent premature LH surges [10-13]. A significant proportion of patients demonstrate an insufficient endogenous LH concentration during ovarian stimulation. However, these patients cannot be distinguished before ovarian stimulation using baseline characteristics alone. For these patients, with either traditional fixed or flexible GnRH antagonist protocols, administration of an antagonist might reduce LH activity further and lead to poor reproductive outcomes [14]. Hence, these patients may not require antagonist supplementation [16,17]. However, there are no clear guidelines regarding the use of $\mathrm{GnRH}$ antagonists based on serum $\mathrm{LH}$ concentrations during ovarian stimulation.

Our previous retrospective study proved that the LH concentration can be used as an indicator for the timing and dosage of antagonist supplementation. Among women who received GnRH antagonists during ovarian stimulation, reproductive outcomes were similar, irrespective of whether the highest LH concentration (LHmax) was $\geq 4 \mathrm{IU} / \mathrm{L}$ or $<4 \mathrm{IU} / \mathrm{L}$. Conversely, patients with a sustained low $\mathrm{LH}$ concentration (LHmax of $<4 \mathrm{IU} / \mathrm{L}$ ) during ovarian stimulation might not require antagonist administration. In fact, in these patients, antagonist administration can adversely affect reproductive outcomes [17]. To further confirm our results, a randomized controlled trial was performed to prospectively compare the efficacy of a novel LH-based treatment regimen with a traditional flexible GnRH antagonist protocol during ovarian stimulation. These results provide clinicians with new information on when to introduce antagonists and the appropriate dosage of $\mathrm{GnRH}$ antagonist.

\section{Methods/design}

\section{Study design}


This study was a multicenter, randomized, controlled, non-inferiority trial that involved patients with normal ovarian responses undergoing in-vitro fertilization (IVF)/intracytoplasmic sperm injection (ICSI). Patients were randomly divided into two groups: the experimental group (stimulated with a LH-based flexible $\mathrm{GnRH}$ antagonist protocol) and the control group (stimulated with a traditional flexible $\mathrm{GnRH}$ antagonist protocol) at a 1:1 ratio on the first day of ovarian stimulation. Figure 1 shows a flowchart of the study design.

\section{Study sites and recruitment procedures}

This clinical trial involved eight hospitals in China. All patients undergoing IVF for the first time at the Center for Reproductive Medicine were included in the study. Physicians will screen patients based on clinical data to assess whether they meet the inclusion criteria. Subjects who are eligible for and who agree to participate in the study are notified and recruited prior to the start of the IVF cycle.

\section{Study population and inclusion/exclusion criteria}

The trial enrolled women who were undergoing their first IVF cycle, and a GnRH antagonist regimen was used for ovarian stimulation. Eligible women met the following inclusion criteria: (1) 23-38 years of age; (2) a spontaneous cycle length of $\geq 21$ days and $\leq 35$ days; (3) a diagnosis of infertility for $>1$ year; (4) an antral follicle count (AFC) of 8-20; (5) a body mass index (BMI) of $\geq 18 \mathrm{~kg} / \mathrm{m}^{2}$ or $\leq 28 \mathrm{~kg} / \mathrm{m}^{2}$.

The exclusion criteria were as follows: (1) a history of unilateral oophorectomy; (2) recurrent spontaneous abortion; (3) a diagnosis of polycystic ovarian syndrome; (4) uterine abnormalities (e.g., submucosal myoma, adenomyosis, uterine scarring, intrauterine adhesion); (5) a chronic medical disease affecting pregnancy outcomes (e.g., diabetes mellitus, hypertension, heart disease, liver dysfunction, renal disease); (6) coagulation dysfunction or a history of deep venous thrombosis. All couples were screened by karyotyping, and those with an abnormal karyotype were excluded.

\section{Randomization}

Randomization was performed by a doctor on the initial day of ovarian stimulation from day 2 of the menstrual cycle. Participants were randomly divided into two groups in a 1:1 ratio and were stratified according to the study site. The randomization scheme was entered into an online central randomization database (www.medresman.org). After randomization, physicians and embryologists were informed about trial group assignment. Patients were randomly assigned to one of two groups: the control group (traditional flexible $\mathrm{GnRH}$ antagonist protocol) or the experimental group (LH-based flexible GnRH antagonist protocol).

\section{Ovarian stimulation protocol}

All patients underwent baseline transvaginal ultrasound and measurement of serum E2, FSH, LH, progesterone $(P)$, and beta-human chorionic gonadotropin ( $\beta-h C G)$ on days $2-3$ of the menstrual cycle. Recombinant FSH (rFSH; Gonal- ${ }^{\circledR}$, Merck, Germany) at a dose of $150-300 \mathrm{IU} /$ day was administered 
according to age, $\mathrm{BMI}, \mathrm{AMH}, \mathrm{AFC}$, and basal serum $\mathrm{FSH}$ concentration. After 5 days of treatment, an ultrasound examination was performed. The gonadotropin dosage was adjusted according to follicle development and serum E2, P, and LH concentrations.

Patients in the control group were administered a GnRH antagonist (cetrorelix acetate, Cetrotide ${ }^{\circledR}$, Merck, Germany) at a dose of $0.25 \mathrm{mg} /$ day, which was initiated when at least one follicle was $14 \mathrm{~mm}$ in size or the $\mathrm{E} 2$ concentration was $\geq 300 \mathrm{pg} / \mathrm{ml}$. Treatment was continued until the day of trigger.

Patients in the experimental group were administered antagonist, and the dosage was based on the LH concentration from day 6 of ovarian stimulation. No antagonist was administered if the LH concentration was $\leq 4 \mathrm{IU} / \mathrm{L}$. If the $\mathrm{LH}$ concentration was $>4 \mathrm{IU} / \mathrm{L}$ or $\leq 6 \mathrm{IU} / \mathrm{L}, 0.125 \mathrm{mg}$ of cetrorelix acetate was administered daily for 2 days until the next blood test. If the $\mathrm{LH}$ concentration was $>6 \mathrm{IU} / \mathrm{L}$ or $\leq 10 \mathrm{IU} / \mathrm{L}$, $0.25 \mathrm{mg}$ of cetrorelix acetate was administered daily for 2 days. If the $\mathrm{LH}$ concentration was $>10 \mathrm{IU} / \mathrm{L}$ or $\leq 15 \mathrm{IU} / \mathrm{L}, 0.375 \mathrm{mg}$ of cetrorelix acetate was administered daily for 1 day. If the LH concentration was $>15 \mathrm{IU} / \mathrm{L}, 0.5 \mathrm{mg}$ of cetrorelix acetate was administered for 1 day. Whether or not antagonist co-treatment was administered depended on an LH concentration of $>4 \mathrm{IU} / \mathrm{L}$ until the day of trigger.

\section{Oocyte retrieval, IVF, and embryo culture}

Final oocyte maturation is induced when at least three follicles reach $\geq 18 \mathrm{~mm}$ in mean diameter using 2000-3000 IU of hCG and 0.2 mg of triptorelin (Decapeptyl ${ }^{\circledR}$, Ipsen, France). Serum LH, E2, and $\mathrm{P}$ concentrations were measured, and transvaginal ultrasound-guided oocyte retrieval was performed 35-36 $\mathrm{h}$ after the trigger injection. Embryo transfer (ET) is usually performed at the cleavage stage, three days after fertilization, with two of the highest quality embryos. The remaining embryos are cultured for two or three more days, and good quality blastocysts are vitrified. Fresh ET was cancelled if patients were at risk of ovarian hyperstimulation syndrome (OHSS), had an unfavourable endometrium (endometrial thickness of $\leq 6 \mathrm{~mm}$ or $\geq 16 \mathrm{~mm}$, cavity fluid, or endometrial polyps), had a P concentration of $\geq 1.5 \mathrm{ng} / \mathrm{ml}$ on the day of hCG trigger, or if no embryo was present. For frozen ET, the endometrium was prepared using either a natural cycle regimen or an artificial cycle regimen based on the physicianof decision.

\section{Outcome measurement}

The primary outcome measure was cumulative ongoing pregnancy rate per cycle. Ongoing pregnancy was defined as a gestational period of more than 12 weeks and fetal heart activity on ultrasound.

Secondary outcomes were high-quality embryo rate, clinical pregnancy rate, and cancellation rate. Moreover, differences in cost-effectiveness and adverse events were evaluated. High-quality embryos were defined by two criteria: the number of cells in the embryo and their appearance under a high-power microscope. Typically, high-quality embryos on day 3 contained 7 to 9 cells with moderate or no fragmentation. Clinical pregnancy rate was defined as the presence of a gestational sac at 6-7 weeks of gestation when visualized by transvaginal ultrasound. Cancellation rate was defined as the number of 
cycles with no embryo for transfer divided by the number of ovum pick-up cycles. Moderate OHSS was diagnosed when ovarian enlargement of $>5 \mathrm{~cm}$ and $<12 \mathrm{~cm}$ was observed and when ultrasonographic ascites was present with or without nausea, vomiting, and/or diarrhea. Severe OHSS was diagnosed when ovarian enlargement of $\geq 12 \mathrm{~cm}$ was observed and when there was clinical evidence of ascites and/or hydrothorax or breathing difficulties with or without hemoconcentration, severe hypoproteinemia, abnormal liver function, coagulation abnormalities, or diminished renal function.

\section{Study assessment}

Screening and baseline assessments included an evaluation of the inclusion and exclusion criteria, study recruitment, and informed consent processes. All patients were evaluated on the day of ovarian stimulation from day 2 of the menstrual cycle. Treatment phase assessments included blood and ultrasound monitoring of follicular development during ovarian stimulation. Hormone analyses were performed during ovarian stimulation as follows: (1) on the day of stimulation; (2) 4-5 days after stimulation initiation; (3) on the day of trigger; (4) 2 days after egg retrieval.

A pregnancy test was performed 12-14 days after ET to confirm pregnancy. In the case of biochemical pregnancy, vaginal ultrasound was performed 6-7 weeks after transplantation to confirm clinical pregnancy. Pregnancy that proceeded beyond 12 weeks of gestation was defined as an ongoing pregnancy. Pregnancy complications (e.g., ectopic pregnancy, OHSS, miscarriage, gestational trophoblastic disease) will be evaluated by inspecting medical records.

\section{Data analysis}

\section{Sample size calculation}

The sample size calculation was based on the cumulative ongoing pregnancy rate. The cumulative ongoing pregnancy rate per cycle in women with normal ovarian responses was approximately $70 \%$ in our retrospective clinical database. Considering that a non-inferiority threshold should retain $80 \%$ of the clinical effect of a control treatment, a minimum clinical difference of $14 \%$ (one-sided: $a, 2.5 \% ; \beta, 20 \%$ ) and a total of 338 patients were needed. Anticipating a $10 \%$ drop-out rate, the total number of patients required was 372 .

\section{Data management}

This trial is registered at clinicaltrials.gov (ChiCTR1800018077). A clinical trial electronic case report form (http://www.clinicaltrialecrf.org) was used to record and deposit patient data to manage the data, monitor the process, and promote research transparency. The study protocol (7 December 2018, version 1.0), operational and procedural manuals, case report forms, informational brochures, and informed consent forms were reviewed and approved by the eight participating hospitals.

\section{Data analysis plan}


Intergroup differences in demographic variables and baseline information were compared before the beginning of the study. Continuous data were analyzed using a two-sample t-test or Wilcoxon's rank-sum test, while categorical data were analyzed using the Chi-squared test or Fisher's exact test.

The primary analysis used an intention-to-treat analysis approach to examine differences in the cumulative ongoing pregnancy rate between the two groups using the Chi-squared test. The relative risk and $95 \%$ confidence interval were calculated. The Chi-squared test or Fisher's exact test was used to analyze secondary efficacy parameters and safety parameters (e.g., clinical pregnancy rate, OHSS rate, cancellation rate).

An independent-samples t-test was used for continuous variables with a normal distribution, and the Mann-Whitney U was used for data with a non-normal distribution. A P value of $<0.05$ (two-sided) was considered statistically significant.

\section{Missing data and dropouts}

Patients who dropped out of the study for whatever reason could still undergo IVF treatment without adversely affecting their cycle. Other outcome variables may have missing data due to missed patient visits.

The flowchart of this study is presented in Fig. 1, and the SPIRIT checklist is included as Fig. 2.

\section{Discussion}

This study aimed to evaluate the efficacy of a LH-based flexible GnRH antagonist protocol in women with a normal ovarian response. Moreover, we aimed to further determine whether LH could be used as an indicator for the timing and dose of antagonist administration with the $\mathrm{GnRH}$ antagonist protocol. We planned to enroll 372 subjects from eight academic IVF centers in China. Patient enrollment began on August 29,2018 . The results of this multicenter randomized trial will provide strong evidence for a $\mathrm{LH}$ based flexible $\mathrm{GnRH}$ antagonist protocol during ovarian stimulation in patients with normal ovarian responses.

This study is a multicenter, randomized, prospective, parallel, non-inferiority study. A threshold concentration of $4 \mathrm{IU} / \mathrm{L}$ of serum LH was used as the cut-off value to determine whether a $\mathrm{GnRH}$ antagonist should be administered. This threshold is based on our previous study of frequent $\mathrm{LH}$ measurements during ovarian stimulation [17]. Previously, we found that most patients with a low LH concentration ( $<4 \mathrm{IU} / \mathrm{L}$ ) throughout ovarian stimulation had no $\mathrm{LH}$ surge. Considering that administration of a $\mathrm{GnRH}$ antagonist would further reduce the $\mathrm{LH}$ concentration, we decided to stimulate patients with $\mathrm{LH}$ at a concentration of $<4 \mathrm{IU} / \mathrm{L}$ without antagonist co-treatment. If our hypothesis proved to be true, a new protocol could be established to control the LH concentration more effectively and potentially improve the effectiveness of IVF treatment, especially in patients with low LH concentrations during ovarian stimulation. 
The majority of patients had sufficient endogenous LH to successfully maintain follicular development and oocyte maturation. However, a significant proportion of patients had an insufficient endogenous LH concentration. Poor pregnancy outcomes were observed in patients who had a continuously low LH concentration and in patients who experienced a sharp decrease in $\mathrm{LH}$ concentration during follicular development from baseline [3-4,18-20]. A relative reduction in $\mathrm{LH}$ concentration in the mid-follicle during GnRH agonist cycles results in a lower live birth rate [21-24]. Studies have shown that when the serum LH concentration on trigger day is lower than one-third of the baseline concentration, pregnancy and implantation rates are significantly reduced [25]. For patients with endogenous LH deficiency, a regimen without antagonists would be more beneficial. Therefore, we implemented this randomized controlled trial to illustrate that LH can be used as an indicator for antagonist administration with a $\mathrm{GnRH}$ antagonist protocol among women undergoing ovarian stimulation for IVF/ICSI. This scheme can be applied to all patients, but is more beneficial for patients with insufficient endogenous LH concentrations. We hope to complete data collection and analysis in order to provide recommendations for the choice of protocol. The data will provide us with a new perspective on the administration of antagonist with a $\mathrm{GnRH}$ antagonist protocol.

\section{Trial status}

The protocol version number and date冈version 1.0, 7 December 2018. The study was conceived and designed in 2017. Enrollment began in 2018 and is expected to end in December 2020. At the time of manuscript preparation, more than 200 subjects had been enrolled. Enrollment in this study was ongoing at the time of manuscript submission.

\section{Abbreviations}

AFC: Antral follicle count; AMH: Anti-Müllerian hormone; ART: Assisted reproductive technology; BMI: Body mass index; CONSORT: Consolidated Standards of Reporting Trials; E2: Estradiol; ET: Embryo transfer; FSH: Follicle-stimulating hormone;Gn: Gonadotropin; GnRH: Gonadotropin-releasing hormone; hCG: Human chorionic gonadotropin; ICSI: Intracytoplasmic sperm injection; IVF: In vitro fertilization; OHSS: Ovarian hyperstimulation syndrome; P: progesterone; RCT: Randomized controlled trial; SPIRIT: Standard Protocol Items: Recommendations for Interventional Trials.

\section{Declarations}

This funding source had no role in the design of this study and will not have any role during its execution, analyses, interpretation of the data, or decision to submit results.

\section{Acknowledgments}

We thank all subjects for their voluntary participation in this study and physicians at all recruiting sites. 


\section{Funding}

This study was supported by 1351 Talent training Program of Beijing Chao-Yang Hospital (CYXX-201720, CYMY-2017-21); Capital Health Development Scientific Research Project (Independent Innovation区 2020-1-2039); Beijing Health Promotion Foundation (2019-09-05); 2018 Fertility Research Program of Young and Middle-aged Physicians-China Health Promotion Foundation.

\section{Availability of data and materials}

The datasets generated during the current study are available from the corresponding author on reasonable request.

\section{Authors' contributions}

YSL were involved in drafting of the manuscript. YL were involved in the study concept and design. SL were involved in the study concept and design and in revision of the manuscript. All authors read and approved the final manuscript.

\section{Ethics approval and consent to participate}

Ethics approval has been sought from the Ethics Committee at the Reproductive medicine center of Beijing Chao-Yang hospital. Written informed consent is obtained from the couple before screening.

\section{Consent for publication}

Not applicable.

\section{Competing interests}

The authors declare that they have no competing interests.

\section{Disclaimer}

The funding bodies had no role in the design of the study and collection, analysis, and interpretation of data and in writing the manuscript.

\section{Author details}

Medical Center for Human Reproduction, Beijing Chao-Yang Hospital, Capital Medical University, 8 Gongren Tiyuchang Nanlu, Chaoyang District, Beijing, 100020, China

\section{References}

1. Depalo R, Trerotoli P, Chincoli A, Vacca MP, Lamanna G, Cicinelli E. Endogenous luteinizing hormone concentration and IVF outcome during ovarian stimulation in fixed versus flexible GnRH antagonist 
protocols: An RCT. Int J Reprod Biomed (Yazd). 2018;16:175-182.

2. Hopkisson J. European Practice in Gynaecology and Obstetrics: Ovulation induction. The Obstetrician and Gynaecologist. 2005;7: 66-66.

3. Hemsey G, O'Brien F, O'Dea L. Objective evidence of LH-dependence in women with profound LH and FSH deficiency. Fertil Steril. 2001;76:208.

4. Balasch J, Miró F, Burzaco I , Casamitjana R, Civico S, Ballescá JL, et al. The role of luteinizing hormone in human follicle development and oocyte fertility: evidence from in-vitro fertilization in a woman with long-standing hypogonadotrophic hypogonadism and using recombinant human follicle stimulating hormone. Hum Reprod. 1995;10:1678-83.

5. O'Dea L, O'Brien F, Currie K, Hemsey G. Follicular development induced by recombinant luteinizing hormone ( $\mathrm{LH})$ and follicle-stimulating hormone $(\mathrm{FSH})$ in anovulatory women with $\mathrm{LH}$ and FSH deficiency: evidence of a threshold effect. Curr Med Res Opin. 2008;24:2785-93.

6. Raju GA, Chavan R, Deenadayal M, Gunasheela D, Gutgutia R, Haripriya G, et al. Luteinizing hormone and follicle stimulating hormone synergy: A review of role in controlled ovarian hyper-stimulation. J Hum Reprod Sci. 2013;6:227-34.

7. Raju G A R, Teng S C, Kavitha P, Lakshmi B, Ravikrishna C. Combination of recombinant follicle stimulating hormone with human menopausal gonadotrophin or recombinant luteinizing hormone in a long gonadotrophin-releasing hormone agonist protocol: a retrospective study. Reprod Med Biol. 2012;11:129-33.

8. Pezzuto A, Ferrari B, Coppola F, Nardelli GB. LH supplementation in down-regulated women undergoing assisted reproduction with baseline low serum LH levels. Gynecol Endocrinol. 2010;26:118-24.

9. Loumaye E, Engrand P, Shoham Z, Hillier SG, Baird DT. Clinical evidence for an LH 'ceiling' effect induced by administration of recombinant human LH during the late follicular phase of stimulated cycles in World Health Organization type I and type II anovulation. Hum Reprod. 2003;18:314-22.

10. Massin N. New stimulation regimens: endogenous and exogenous progesterone use to block the LH surge during ovarian stimulation for IVF. Hum Reprod Update. 2017;23:211-20.

11. Lahoud R, Al-Jefout M, Tyler J, Ryan J, Driscoll G. A relative reduction in mid-follicular LH concentrations during GnRH agonist IVF/ICSI cycles leads to lower live birth rates. Hum Reprod. 2006;21:2645-9.

12. Ruvolo G, Bosco L, Pane A, Morici G, Cittadini E, Roccheri MC. Lower apoptosis rate in human cumulus cells after administration of recombinant luteinizing hormone to women undergoing ovarian stimulation for in vitro fertilization procedures. Fertil Steril. 2007;87:542-6. 
13. Lahoud R, Jefout M A, Tyler J, Ryan J, Driscoll G . A relative reduction in mid-follicular LH concentrations during GnRH agonist IVF/ICSI cycles leads to lower live birth rates. Human Reproduction. 2006;21:2645-9.

14. Haas J, Ophir L, Barzilay E, Machtinger R, Yung Y, Orvieto R, et al. Standard human chorionic gonadotropin versus double trigger for final oocyte maturation results in different granulosa cells gene expressions: a pilot study. Fertil Steril. 2016;106:653-9.

15. Huang Q, Niu Y, Xu L, Niu Y, Xu L, Chen B, et al. Relationship between a low ratio of serum estradiol to follicle number and fertility treatment outcomes. Medicine. 2018;97:e12017.

16. Yanaihara A, Yorimitsu T, Motoyama $\mathrm{H}$, et al. The decrease of serum luteinizing hormone level by a gonadotropin-releasing hormone antagonist following the mild IVF stimulation protocol for IVF and its clinical outcome. J Assist Reprod Genet. 2008;25:115-8.

17. Liu M, Li S, L L, Wang P , Li H, Li Y. LH Levels May Be Used as an Indicator for the Time of Antagonist Administration in GnRH Antagonist Protocols-A Proof-Of-Concept Study. Front Endocrinol. 2019;10:67.

18. Costello M F, Misso M L, Balen A H, Boyle J, Devoto L, Garad R M, et al.. Evidence summaries and recommendations from the international evidence-based guideline for the assessment and management of polycystic ovary syndrome: assessment and treatment of infertility. Hum Reprod Open. 2019;2019: hoy021.

19. Marca AL, Grisendi V, Giulini S, Argento C, Tirelli A, Dondi G, et al. Individualization of the FSH starting dose in IVF/ICSI cycles using the antral follicle count. Journal of Ovarian Research. 2013;6:11.

20. Benmachiche A, Benbouhedja S, Zoghmar A, Humaidan P. Low LH Level on the Day of GnRH Agonist Trigger Is Associated With Reduced Ongoing Pregnancy and Live Birth Rates and Increased Early Miscarriage Rates Following IVF/ICSI Treatment and Fresh Embryo Transfer. Front Endocrinol. 2019;10:639.

21. Boularak A, Humaidan P. Impact of mid-luteal phase GnRH agonist administration on reproductive outcomes in GnRH agonist-trigger: a randomized controlled trial. Front Endocrinol. 2017;8:124.

22. Connell MT, Patounakis G, Healy MW, DeCherney AH, Devine K, Widra E, et al. Is the effect of premature elevated progesterone augmented by human chorionic gonadotropin versus gonadotropinreleasing hormone agonist trigger? Fertil Steril. 2016;106:584-9.

23. Venetis CA, Kolibianakis EM, Bosdou JK, Tarlatzis BC. Progesterone elevation and probability of pregnancy after IVF: a systematic review and meta-analysis of over 60000 cycles. Hum Reprod Update. 2013;19:433-57.

24. Doody KJ, Devroey P, Leader A, Witjes H, Mannaerts BM. No association between endogenous LH and pregnancy in a GnRH antagonist protocol: part I, corifollitropin alfa. Reprod Biomed Online. 2011;23:457- 
65.

25. Bildik G, Akin N, Seyhan A, Esmaeilian Y, Yakin K, Keles I, et al. Luteal granulosa cells from natural cycles are more capable of maintaining their viability, steroidogenic activity and LH receptor expression than those of stimulated IVF cycles. Hum Reprod. 2019;34:345-55.

\section{Figures}




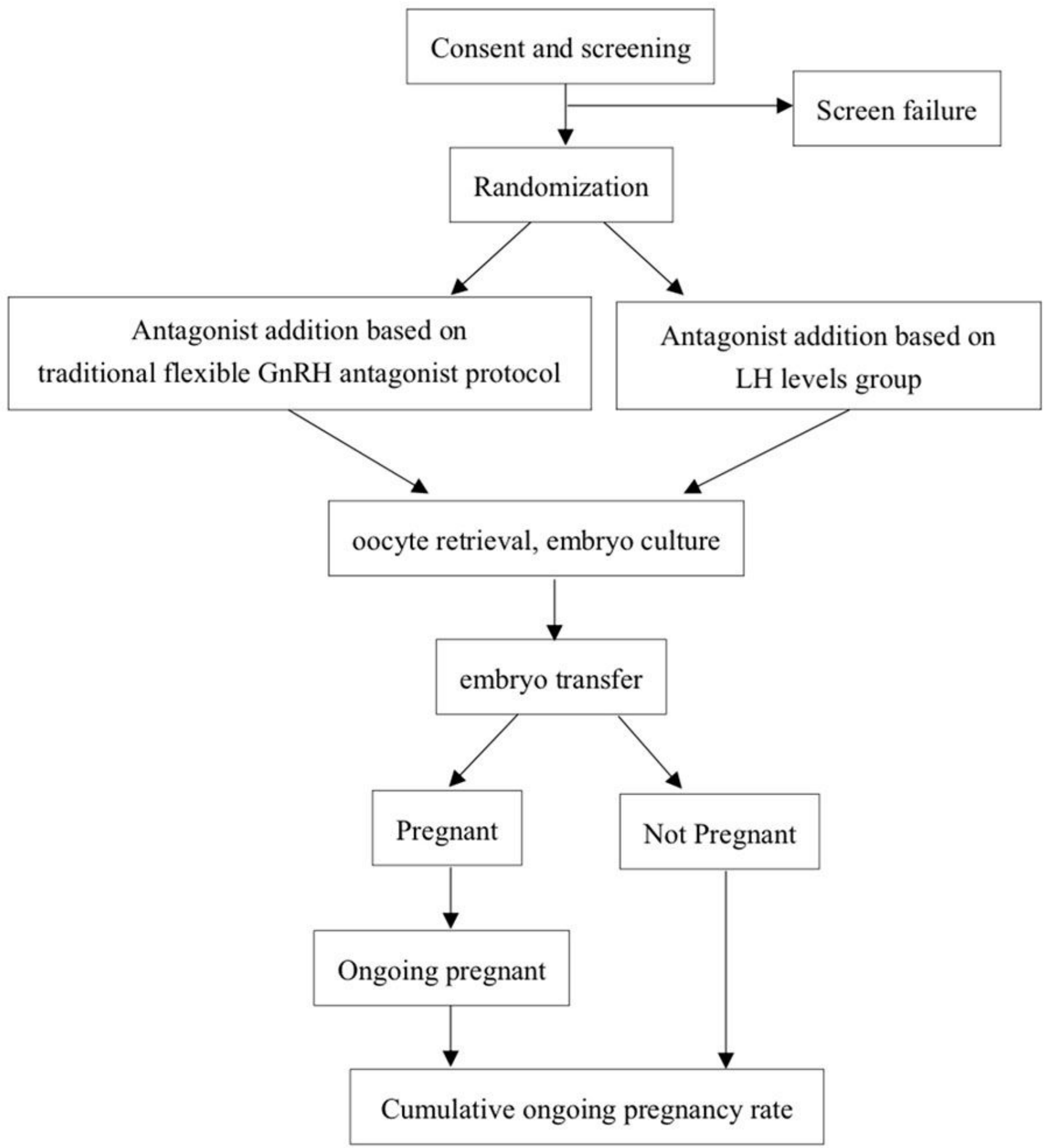

Figure 1

flowchart of the study design.

\section{Supplementary Files}

This is a list of supplementary files associated with this preprint. Click to download. 
- Additionalfile1.pdf

- RCT1SPIRITChecklistforrandomisedstudies.doc 